\title{
Aproximación a un estudio sociolingüístico del habla de Ceuta
}

\author{
Teresa-G. Sibón Macarro
}

\section{Introducción. Objetivos y metodología}

Un territorio puente entre dos continentes, europeo y africano, ha sido la zona elegida para nuestro muestreo y análisis sociolingüístico ${ }^{1}$. Y lo que le hace peculiar a esta comunidad lingüística ${ }^{2}$ es precisamente el haber sido lugar de paso obligado de las expansiones de otros pueblos y culturas, sociedades y tradiciones a lo largo de distintas épocas.

Para un curioso observador, resulta delicioso y muy atrayente contemplar desde cualquier rincón de esta ciudad la enorme variedad de culturas y su mestizaje, que da como resultado una sugestiva síntesis que se plasma en el ambiente ceutí. Si esto sucede con el ensamblaje de distintas culturas, cómo será en su medio común de expresión: la riqueza y variedad de usos lingüísticos.

En nuestro plan de trabajo, comenzamos con una descripción del contexto geográfico, político-social, y humano, para, desde esa situación, aproximamos a una valoración sociolinguiística más coherente ${ }^{3}$. Y, en el anexo de este trabajo, recogemos los resultados de las encưestas sintetizadas ${ }^{4}$ y con el cuadro de porcentajes.

Para lograr este objetivo hemos seguido los siguientes modelos de encuestas:

I a) ¿Qué lengua habla?

b) ¿Cree que en Andalucía se habla bien o mal?

\footnotetext{
${ }^{1}$ Quisiera agradecer a M Mesús y José Luis Sibón Macarro su valiosísima colaboración en la labor de encuestas y recopilación de datos entre los informantes ceutíes.

${ }^{2}$ Definición: Labov, 1983; p. 54. Gumperz Bennet, 1981; p. 135.

${ }^{3}$ Según los tres pasos que propone Labov, 1983; pp. 177-178.

${ }^{4}$ Demasiado extenso si transcribiéramos una por una todas las encuestas.
} 
c) En España, ¿quiénes cree que hablan de forma más correcta?

d) Y en Andalucía, ¿quiénes cree que hablan mejor, de forma más correcta?

e) Si tuviera que hablar en público, en TV o radio, ¿lo haría en andaluz?

II a) ¿Cómo llamaría a su forma de hablar?

b) ¿La considera correcta, o incorrecta (su forma de hablar)?

c) ¿Dónde cree que se habla más correctamente?

d) ¿Y entre las provincias andaluzas?

e) Si saliera de Andalucía, ¿cambiaría su forma de hablar? (sí o no)

(Las cuestiones IIc y IId se unifican en las conclusiones, pues, salvo alguna excepción, las respuestas coinciden, -y el análisis de tales excepciones no es relevante).

Un dato curioso: llamaba la atención el cambio de actitud que mostraban los informantes (desconfianza u orgullo) al pasar del Modelo I al Modelo II de las encuestas. (Como consecuencia de esto, incluso algunas personas se negaron a continuar con el segundo modelo, y no conseguimos saber las causas).

Otro dato de sustancial relevancia en la configuración de un informe sociolingüístico completo son los siguientes:

Edad del informante.

Sexo.

Zona donde vivía.

Si estudia o trabaja.

Con respecto a la edad, tuvimos que admitir una aproximada de alguna persona de $3^{a}$ generación. Y del último punto que nos sitúa al encuestado, hay que decir que el sector más asequible a nuestro trabajo era el comercial; además, de que desde la mayoría de edad es más probable esta segunda situación.

En total hemos contado con la colaboración de cien personas encuestadas, de las cuales eran sesenta y dos hombres $(\mathrm{H})$ y treinta y ocho mujeres $(\mathrm{M})$. Esto se ha hecho así con la intención de recoger la misma proporción diferencial que se da entre la población ceutí, o sea, mayoría hombres.

Y para percibirunas posibles matizaciones según la generación a la que pertenecían, distinguimos tres grupos generacionales, cuyo centro-eje es el formado por personas de veintiséis a cuarenta y cinco años.

Entre hombres y mujeres, un cuadro de estos datos sería el siguiente:

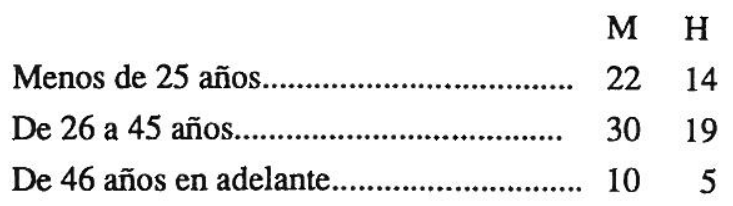


A partir de los datos aportados por el corpus de encuestados, analizaremos el contacto y proceso de identificación, el mantenimiento o desplazamiento ${ }^{5}$ de tal o cual uso en la conciencia del hablante. De este modo, llegaremos a conocer las preferencias de los ceutíes o caballas al dar un nombre a su modo de hablar, y la valoración que de esta hacen; descubriremos su inconsciente actitud de lealtad u orgullo-en su caso, como anunciamos más arriba-, y los criterios de autoridad que lo sostentan ${ }^{6}$.

Los encuestados son casi en su totalidad de Ceuta, con un mínimo de ascendencia ceutí de dos o tres generaciones, (la minoría no ceutí corresponde a dos almerienses, tres madrileños, un marroquí, y cuatro gaditanos que llevaban cuatro años viviendo allá).

\section{Ceuta, su entorno}

Ciudad de soberanía española desde 1688 (Tratado entre España y Portugal); situada en el litoral del Norte de África, frente al Peñón de Gibraltar, rodeada por el territorio del reino de Marruecos. Tiene una extensión de diecinueve kilómetros cuadrados y una población que oscila los ochenta y cinco mil habitantes (1968).

La primera noticia escrita y fidedigna de Ceuta, relacionada ya con la Península, es de hace unos 2.500 años con la primera descripción del Estrecho de Hércules y de Abyla, el solar ceutí.

La historia es par a Ceuta y la Península; fenicios, púnicos o cartagineses; romanos o la dominación árabe; los colonialismos,... hasta hoy día, la base de Ceuta ha sido utilizada para dominar ambos márgenes del Estrecho -apoyándose en la Columnas de Hércules, Abyla y Calpe, que señalaban el confín del mundo conocido ${ }^{7}$.

Por dar un dato más contemporáneo al nuestro, y que nos ayudaría a situarnos en el punto de vista de los ceutíes, recordaremos la Guerra de Africa de 1859-60 o los Tratados Internacionales de 1912, por los que se inicia el Protectorado español en ese pequeño territorio del Norte de África ${ }^{8}$.

Es conveniente conocer las condiciones del territorio para explicarnos las condiciones de factor humano, político, social, cultural que han ido dejando sús rasgos peculiares a su paso por esas tierras.

La ciudad está asentada sobre la pequeña península de Almina, coronada por el Monte Hacho (195 m.) y termina a orillas del estrecho de Gibraltar en Punta Almina, y continúa sobre el istmo que une la Península al continente africano?.

${ }^{5}$ Es paradójico el comprobar la poderosa fuerza del habla de la clase culta o dominante, y, sin embargo, quien lleva la voz cantante-nunca mejor dicho-en las transformaciones y evolución es el pueblo.

${ }^{6}$ Hagège, 1987; p. 144.

${ }^{7}$ Ciudad de Ceuta, Edit. Imo. Ayuntamiento Ceuta, 1981; p. 9.

${ }^{8}$ Idem, p. 9.

9 «La Historia de la Ciudad de Ceuta, por ser un compendio y trasunto fiel de la Historia de España, con las mismas dominaciones, con idénticas vicisitudes y avatares, es un caso singular de constante vocación ibérica, secularmente vinculada al contexto peninsular desde el mismo día de su nacimiento». Idem, p. 9. 
El territorio de la ciudad está accidentado por diversas alturas derivadas de la Sierras de Ányena y de Bullones, el punto más destacado hacia el Estrecho es .YabalMusa. Existe una zona o Campo Neutral, donde están instalados los servicio aduaneros de la frontera con Marruecos.

Su clima es mediterráneo, con claro matiz atlántico; con un cultivo característico de estas condiciones: viñedo. Y el punto fuerte de su economía se basa en el comercio. Este se mantiene a través de su amplio puerto, lugar de recalaje obligado en la entrada al Mediterráneo. Junto a esto, mantiene su próspera actividad pesquera, que ha permitido el desarrollo de una pequeña industria pesquera.

Constituye un núcleo militar importante con elevada guamición, que es otra fuente de ingresos para la población ceutí.

Desde el punto de vista administrativo y judicial, depende de la provincia de Cádiz -una de las claves del problema de mantenimiento peninsular, pudiendo ser más beneficiosos traer el suministro de Marruecos. Con todo, su problema radica en la debilidad de recursos locales y el crecimiento de población. Cuestión que obliga al gobierno a fuertes inversiones en ampliación y mejora del puerto y obras de urbanización.

\section{Ceuta, datos de población}

Hoy día, los datos proporcionados por el C.E.P. ${ }^{10}$ sobre el total de habitantes son los siguientes:

1ํ Abril 1986. De derecho 65.151 habit.

De hecho 71.403 habit.

$1^{\circ}$ Abril 1989. De derecho. 68.014 habit.

De hecho 74.500 habit. $^{11}$

La diferencia entre las cifras correspondientes a «De derecho» $\mathrm{y}$ «De hecho» se debe a la población flotante; o sea, ese aumento de uno a otro se debe a personas cuya estancia en Ceuta es accidental, comerciantes ambulantes, militares (los que cumplen el servicio militar, o los destinados a esta ciudad -de distinta temporalidad), turistas que permanecen una temporada.

Según los datos proporcionados por el C.E.P. de Ceuta, hay una diferencia importante entre la población de hecho y la de derecho que, como adelantamos antes, correspondía hipotéticamente a los reclutas y otros miembros del Ejército (con una estancia de menor y mayor temporalidad, respectivamente), comerciantes y turistas.

Quisiéramos transcribir algunos datos estadísticos sobre las distintas razas y creencias que se ensamblan entre la población, pero al solicitarlas al C.E.P., lo

${ }^{10}$ Centro Estadística Población de Ceuta (Tf́no.: 956512608 )

${ }^{11}$ No podían facilitarnos una cifra precisa porque aún no la tenían oficialmente. 
denegaron alegando la posible inconstitucionalidad de estas preguntas. De ahí que no podamos obtener dato preciso sobre los magrebíes, indios o los hindúes, los hebreos. Sí nos han podido proporcionar una cifra aproximada de las personas (esta cantidad es la moda sacada entre la fluctuación de las corrientes de población según la estación del año). Y el mismo valor tiene la referida al colectivo hindú y hebreo, unas 500 personas ${ }^{12}$.

Además de lo señalado, la gente joven que marcha a estudiar o a trabajar donde le depare mejor futuro, es causa de baja de población en Otoño e Invierno. Ceuta no tiene la posibilidad de ofrecer distintas carreras o ramas a sus habitantes; a sus habitantes les corresponde la Universidad de Cádiz.

Esta ciudad norteafricana es también punto terminal de continuos viajes de extranjeros o de peninsulares, pero no para residir de forma prolongada, sinopor turismo o para compras. Las fechas altas o temporadas más fuertes en este aspecto son Navidades, Semana Santa, y en el Verano (por el turismo en Costa Sol y el Golfo de Cádiz), aunque -según informes del C.E.P.- se mantiene una básica afluencia constante durante todo el año y en menor número.

Entre los datos que nos facilitaron, los tantos por ciento de población, con la división en varones y mujeres, son:

En 1986:

$$
\begin{aligned}
\text { De derecho................. } & \text { V. } 49,13 \% \\
& \text { M. } 50,87 \% \\
\text { De hecho.................. } & \text { V. } 53,29 \% \\
& \text { M. } 46,61 \%
\end{aligned}
$$

En 1989:

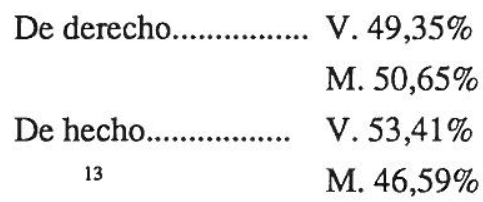

(En estos porcentajes están incluidos españoles, magrebíes, hindúes, hebreos).

La diferencia inversa entre V y M, y entre «De hecho» y «De derecho», principalmente encuentra gran parte de su explicación en el destino militar de nuevos reclutas. No se puede calcular nunca con precisión esta población flotante, porque no

\footnotetext{
${ }^{12} \mathrm{La}$ Delegación de Gobiemo ha redactado, como en otras ocasiones, un informe sobre estas cuestiones, y uno de los apartados trataba de la 'Operación tránsito del Estrecho'. Esto es, argelinos y marroquíes en su recorrido del N (Europa) al S (su tierra), y viceversa. Así sucede al principio del verano, y al retomo cuando este finaliza.

${ }^{13}$ Las fechas 'De hecho' están calculadas aproximadamente porque el C.E.P. no estaba facultado a darlas oficialmente. En los tantos por ciento están incluidos españoles, magrebíes, hindúes, hebreos.
} 
se prevén los cambios de patrulla -según nos razonaba un alto mando militar de allá. A los oficiales y suboficiales se les ha considerado dentro de la población «De derecho», ya que necesitan un certificado de residencia fuera de la Península.

Un dato importante es el matiz político en relación con el origen del individuo; es decir, si pertenece a una primera, segunda o tercera generación en tierra ceutí, y si conoce o vivió los conflictos con los antiguas colonias españolas ${ }^{14}$.

Analicemos las influencias desde los medios de comunicación; la Televisión que ven los españoles residentes conecta normalmente con la Península, o con cualquier emisión inglesa o marroquí, pero aún no tienen su propio canal -donde quizá captáramos mejor la intersección entre culturas y lenguas. En radio sí localizamos frecuencias de la tierra. En la prensa escrita, como en Televisión, también tiene un puesto prioritario la de edición peninsular, sobre toda cara a la riqueza de información que proporciona esta frente a la ceutí. Pero, en definitiva, este factor influye sobre la actualización del lenguaje.

Las escuelas cuentan con alumnos que proceden de variadas familias ${ }^{15}$; y parecerá esta aclaración un dato indiferente, pero no es así -al menos en esta ciudad. Dicho con otras palabras, sabemos por experiencia que los primeros pasos en el aprendizaje de una lengua están en el hogar, pronto lo que la criatura veía establecido de una determinada manera se encuentra con otras modalidades que incluso no entiende (para comprobar esto bastaría el hecho de presenciar la salida de la escuela).

La sociedad es absolutamente cosmopolita y, por la antigüedad de esta convivencia entre habitantes tan distintos, no se percibe mentalidad especial de filtro o impermeabilidad de 'préstamos' e influencias. A pesar de este rasgo general, sí es notorio el subconsciente de rechazo hacia lo que denominaríamos convencionalmente 'lo moro'. Esto es fruto de la imagen típica tópica, -- tradicional ya, exagerada y falseada--, de la mentalidad del habitante de origen peninsular -peculiar entre los hispano-ceutíes.

Con esto, cada tipo diferencial de población se focaliza en una zona determinada. El recorrido es corto; una guía, la calle Real que la cruza desde el Puerto hasta la zona central. Por el barrio moro, como se conoce allí, no es prudente ir más que de día. Y los lugares de diversión son distintos para residentes españoles a los de otra nacionalidad.

En definitiva, a la fusión sin prejuicios de estas culturas y razas, le corresponde, paradójicamente, una perenne división entre las gentes del magreb y los peninsulares como indicamos más arriba-. Con esto, el ser territorio de paso, además de estratégico -políticamente hablando-, siempre le ha dotado de cualidades peculiares a la historia del lugar y a su gente ${ }^{16}$. Su historia está vinculada a la Península, a la provincia gaditana,

${ }^{14}$ Este punto es importante si se acepta la idea de que unळlengua es reflejo de la cultura, mentalidad y organización social de un pueblo.

${ }^{15}$ En relación con la norma materna. Ropero, 1989; p. 9.

${ }^{16}$ Continuamente se entrecruzan los modos de hablar tradicionales, las hablas de distintos niveles, edades, etc. 
desde el S.XVII ${ }^{17}$. Su economía, descontando el ser foco comercial, no es muy distinta al resto de la zona norte africana. La fuente de cultura es plenamente peninsular en cuanto a institución y enseñanza.

Todo esto influirá directamente en la caracterización sociolingüística consecuencia de la intersección diatópica, diastrática, diafásica en sincronía ${ }^{18}$. Además, no nos detendremos a ver la realización fonológica y fonética, ni matices diferenciales desde el punto de vista gramatical, sino la conciencia local de su modo de hablar, como se deduce de las cuestiones que se plantean en estas encuestas.

Los resultados obtenidos de las encuestas realizadas en Ceuta, se confrontarán, en otro trabajo más amplio que esta resumida aproximación -que concluiremos próximamente-, con los elaborados en otros puntos de Andalucía, en los que se aplicó el mismo método ${ }^{19}$.

Y pretendemos seguir en este trabajo una metodología que, analizando el habla desde fuera y dentro del fenómeno, aporte unos datos y conclusiones con la mayor objetividad que se pueda alcanzar ${ }^{20}$.

\section{Ceuta, variables en su habla}

En el mapa incorporado en el anexo (p. 3) aparecen localizados con puntos las zonas donde realizamos las encuestas ${ }^{21}$. Y ya advertimos más arriba la dificultad de adentramos en lo que se conoce como 'el barrio moro' -del que, por otra parte, podríamos intuir las respuestas. En definitiva, los sectores donde hemos aplicado las encuestas son la zona de la Muralla y el centro urbano.

Una ojeada por la guía telefónica de la ciudad acusa el ensamblaje de pueblos producidos, hecho que procuramos recoger en la labor de selección de informantes -con no pocas controversias.

El nivel cultural es más bien medio, según la mayoría de los informantes. Además, las encuestas se realizaron en invierno, con el propósito de localizar a aquellas personas cuya residencia es más estable; es decir, no contamos con los habitantes que se encuentran estudiando ni trabajando en la Península, que quizá pertenecieran a un nivel cultural superior. $O$ sea, loss casos de informantes que fueran de otro nivel cultural no eran ceutíes con ascendencia también caballa.

A las puntualizaciones anteriores, se suma el hecho de que las personas que permitían ser interrumpidas en su quehacer unos instantes, eran las que se encontraban trabajando en los comercios, las señoras que salían de compras -a las que prácticamente

${ }^{17} \mathrm{La}$ conquista de las plazas costeras por los cristianos, durante las ofensivas hispanas y portuguesas de los SS. XV-XVI, despertó en los marroquíes la conciencia de su decadencia.

${ }^{18}$ Cfr. Lamíquiz, 1982; p. 18, pp. 57-80.

${ }^{19}$ Cfr. Ropero y Pérez Santamaría, 1989.

${ }^{20} \mathrm{Cfr}$. Lamíquiz, 1982; pp. 25-43.

${ }^{21}$ Desde la zona del Puerto hasta la derecha e izquierda de la calle Real. 
se les acompañaba-, y aquellas gentes que, desde cualquier rincón, contemplaban la vida cotidiana de otros -estos eran de la tercera generación en su mayoría.

Por lo que corresponde a los citados dependientes de los comercios, conviene destacar un dato, como un implícito factor de influencia que justificaría cierto orgullo de su modo de hablar; y este dato es la peculiar facilidad que poseen para defenderse en otras lenguas distintas de la suya, según les obliga su misma dedicación profesional.

Otra variable a tener en cuenta es la cuestión de la edad. Así, las personas de tercera generación han sufrido los acontecimientos de la Guerra de África, el Protectorado español, las actuales ayudas francesas; y ni parte de la segunda ni la primera generación pasaron todas esas circunstancias. Estos sucesos indudablemente condicionarán una respuesta.

En cuanto a la variable sexo, un dato curioso es esa cierta lealtad al modo de hablar Peninsular que subyace en las respuestas de los informantes $\mathrm{M}$ frente a los informantes $\mathrm{H}$ (cfr. Anexo), y es posible que tenga bastante relación con los acontecimientos aludidos en el párrafo anterior.

Conviene tener presente la técnica de los cálculos estadísticos y sus porcentajes. Es decir, si de treinta $y$ ocho informantes $M$, un piensa ' $X$ ', supone un $2,63 \%$ del total; y si de sesenta y dos informantes $H$, uno piensa ' $X$ ' resulta un $1,61 \%$. Por esta razón, nuestras consideraciones remitirán a los tantos por ciento del Anexo ${ }^{22}$.

Las denominaciones que dan a su modo de hablar, en Ia, fluctúa entre «Español» y «Castellano», en $\mathrm{H}(51,61 \%)$. Muestra del orgullo al que nos referíamos más arriba es el elegir el término con connotaciones de referencia a Estado, sobre el «Castellano» -usado como sinónimos al aplicarlo al hablar peninsular.

Y, curiosamente, los que se reafirman en su determinación por el «Castellano» en Ila son $\mathrm{H}(7,8 \% \mathrm{M}-17,75 \% \mathrm{H})$, manteniéndose «Español» en equilibrio (10,4\% M $11,3 \%)$.

La valoración psico-sociolingüística ${ }^{23}$ ceutí se recoge en las preguntas Ib y IIb; y, en coherencia con ellas, las Ie y IIe que proporcionan la información sobre la actitud de los ceutíes ante sus usos lingüísticos ${ }^{24}$.

En este sentido, las respuestas al bien o mal de su hablar ${ }^{25}$ se inclinan a considerarla «mal» $(50 \% \mathrm{M}-30,64 \% \mathrm{H})$, pues la comparan con su respuesta anterior: «Español» «Castellano». Pero después de situar al informante en la realidad andaluza, con el Modelo II, parece que la corrección o incorrección de su habla se identifica con validez -o afecta al orgullo personal-, pues se 'palpa' el peso de «Correcto» (55,26\% M-69,35\% H) sobre «Incorrecto» $(31,58 \% \mathrm{M}-16,13 \% \mathrm{H})$. El resto de las respuestas genera un

\footnotetext{
${ }^{22}$ No obstante, conviene confrontar los porcentajes con los números absolutos del Anexo, en las primeras páginas. Y otro punto que quisiera aclarar es el siguiente: cuando el informante quería mantener dos posibilidades en preguntas como Ic-d o IIc-d, se contaban ambas; o sea, en el cálculo estadístico la suma de los tantos por ciento resultan, a veces, mayores de $100 \%$, y esta es su causa.

${ }^{23}$ Ropero (1989), p. 202.

${ }^{24}$ Ropero (1989), p. 204.

${ }^{25}$ Y nos centraremos en los extremos para ser más acorde en la comparación con Ie y Ile, sobre su actitud.
} 
campo intermedio de inseguridad o ambigüedad, que se pierde en respuestas tales como «Normal», «Mejorable», «Según», «Regulan», y los que dudan al emitir su opinión.

En cuanto a la actitud de los ceutíes -globalizando las respuestas de nuestros informantes-, se produce una notoria variación. Véase la diferencia entre le y Ile en los síes y nones referidos a su situación de habla en público:

SI la cambiarían (Ie)................ M 63,16\% - H 61,3\%
NO la cambiarían (IIe).......... M 86,84\% - H 82,26\%

cambio de matiz bastante sorprendente; es la misma cuestión, pero la segunda centra al informante en su contexto real, por lo que el orgullo al juzgar su modo de hablar pesa más en el segundo planteamiento. Y lo más desconcertante, en cierto modo, es que $\mathrm{M}$ en IIe es superior -como si su cambio de actitud estuviera orientada por la ley del péndulo, posibilidad que, por otra parte, corresponde a la psicología femenina.

En cuanto a la Ic-d, el modelo linguístico corresponde a lo esperado y casi tópico; o sea, el Centro peninsular es el poseedor del habla idónea (cfr. Anexo p. 4). En el marco andaluz, se delata un subconsciente condicionado quizá por la pregunta anterior de ese mismo modelo (sobre la posible corrección o incorrección en la actualización de su habla), pues se destaca una variable que excluye cualquier referencia concreta: «Nadie» con un peso fuerte nuevamente entre las mujeres $(15,78 \% \mathrm{M}-11,29 \% \mathrm{H})$-la oposición con «Todos» no la analizamos por su irrelevancia $(1,6 \% \mathrm{H})$.

La respuesta «Ceuta» coincide entre los informantes $\mathrm{M}$ y $\mathrm{H}$ en Id y IIc-d, sin reducirse por ello la consideración al pasar de un Modelo a otro. Sin embargo, crecen los porcentajes de 'dudosos' en comparación con Ic (5,25\% M - 8,06\% Hen Ic $><39,5 \%$ M- 24,19\% en IIc-d).

En conclusión, se percibe un enlace subconsciente con lo peninsular en los datos suministrados en el primer Modelo, rasgo que se pierde o difumina con la aplicación del segundo Modelo. En este el habla ceutí, junto con la gaditana, cobra su personalidad en la consciencia del hablante, y así se corrobora en la cuestión más definitiva, desde nuestro punto de vista: IIe «Si saliera de Andalucía, ¿cambiaría su forma de hablar? (SI o NO)»: NO es la respuesta ceutí con una media superior a las tres cuartas partes del total $(86,84 \% \mathrm{M}-82,26 \% \mathrm{H})$.

\section{Bibliografía}

ALFAYA GONZÁLEZ, J. M. (1985); «La actitud de la prensa marroquí(1956-75) ante la presencia de España en el Norte de África»; Actas de la II Jornada de Cultura Árabe e Islámica (1980), I.H.A.C.M. 1985; (pp. 21-23).

BOSQUE MAUREL, J. y MARÍN, N. (1971); «Ceuta», Madrid, Rialp; GER T. 5, Voz 'Ceuta', (pp. 562-563).

CARBONERO CANO, P. (1982); El habla de Sevilla; Sevilla, Biblioteca de Temas Sevillanos, (cap. V-VI). 
FISHMAN, J. A. (1988); Sociología del lenguaje; Madrid, Cátedra; 3ª edición.

HAGÈGE, C. (1987); La estructura de las lenguas; Madrid, Gredos, (pp. 121-155).

LABOV, W. (1983); Los modelos sociolingüísticos; Madrid, Cátedra.

LAMÍQUIZ, V. ea. (1982); Sociolingüística andaluza; Sevilla, Publicaciones Univ. Sevilla, (pp. 137-146,159-167).

LOPE BLANCH, J. M. (1972); El concepto de prestigio y la norma lingüística del español; México, (pp. 81-47).

MORALES LEZCANO, V. (1976); El colonialismo hispanofrancés en marruecos; Madrid, S. XXI.

NAVARRO ALCALÁ-ZAMORA, P.J. (1981); Sociedad, pueblos y culturas; Barcelona, Aula Abierta Salvat.

ROPERO NÚÑ̃E, M. y Pérez Santamaría, F. J. (1989); Conciencia y actitudes sociolingüísticas en Andalucía, Sevilla. (En vías de publicación por Ilmo. Ayuntamiento de la Ciudad de Sevilla).

VARIOS (1986); El castellano actual en las comunidades bilingües de España; Junta de Castilla y León, Consejería de Educación y Cultura.

VARIOS (1981); Ceuta. La España inédita; Ilmo. Ayuntamiento de Ceuta, Patronato Municipal de Turismo; Madrid, (pp. 13-29).

\section{ANEXO}

Ia. ¿Qué lengua hablas?

$\begin{array}{lccrccc}\text { M. } & \left(1^{\mathrm{a}}\right. & \text { Español } & 8 & / & \text { Castellano } & 6 \\ & \left(2^{\mathrm{a}}\right. & " & 12 & / & " & 7 \\ \left(3^{\mathrm{a}}\right. & " & 3 & / & " & 2\end{array}$

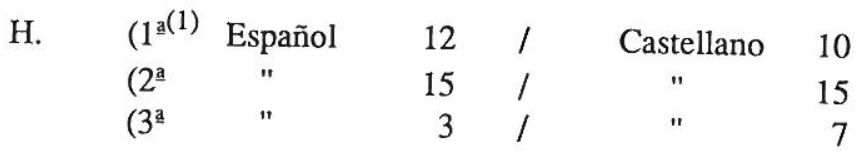

Ib. ¿Crees que en Andalucía se habla: BIEN o MAL?

$\begin{array}{lllllll} & & \text { B } & \text { N } & \text { S } & \text { R } & \text { M } \\ \text { M. } & 1^{\mathrm{a}} & 3 & 1 & \varnothing & 2 & 8 \\ & 2^{\mathrm{a}} & 7 & \varnothing & 2 & 1 & 9 \\ & 3^{\mathrm{a}} & 1 & 2 & \varnothing & \varnothing & 2\end{array}$




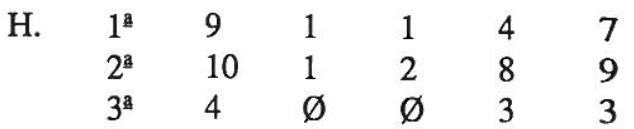

Ic. En España, ¿quiénes cres que hablan mejor, más correctamente?

$\begin{array}{llllllllllllll} & \text { Nd } & \text { Td } & \text { Ln } & \text { Md } & \text { Sl } & \text { Vl } & \text { Ct } & \text { Cs } & \text { Nt } & \text { Ctr } & \text { i? } & \text { And } & \text { Cu } \\ \text { M. } 1^{\mathbf{a}} & \emptyset & \emptyset & \emptyset & 4 & \emptyset & 2 & (2) & 4 & 1 & 2 & \emptyset & \emptyset & \emptyset \\ 2^{\mathbf{a}} & \emptyset & \emptyset & \emptyset & 4 & 1 & 4 & \emptyset & 3 & 1 & 3 & 1 & 2 & \emptyset \\ 3^{\mathbf{a}} & \emptyset & \emptyset & \emptyset & \emptyset & \emptyset & 2 & \emptyset & 2 & \emptyset & \emptyset & 1 & \emptyset & \emptyset\end{array}$

$\begin{array}{llllllllllllllll}\text { H. } & 1^{\underline{a}} & 1 & \emptyset & 1 & 5 & 1 & \emptyset & 1 & 5 & \emptyset & \emptyset & 2 & 2 & 1 & \text { Tol }\end{array}$ $\begin{array}{lllllllllllllll}2^{\mathrm{a}} & \emptyset & 2 & 2 & 4 & \emptyset & 5 & \emptyset & 9 & 1 & 1 & 2 & 2 & 1 & \text { Ext }\end{array}$

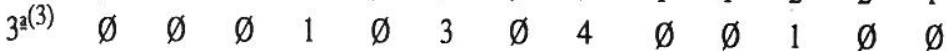

Por orden corresponden a: Nadie, todos (igual), León, Madrid, Salamanca, Valladolid, Cataluña, Castilla, Centro (peninsular), los que «no saben» - «según», Andalucía, Ceuta -no se dicen por gentilicios por las cuatro referencias generales. Los del margen son: La Mancha, Zamora, «parte Sur», Toledo y Extremadura.

Id. Y en Andalucía, ¿quiénes crees que hablan de forma más correcta?

$\begin{array}{llllllllllllll} & \text { Nd } & \text { Td } & \text { Mlg } & \text { Cdz } & \text { Ceu } & \text { Cor } & \text { i? } & \text { Se } & \text { Gr } & \text { Hu } & \text { Jn } & \text { Alm } & \text { Sur } \\ \text { M. } 1^{\mathbf{a}} & 5 & \emptyset & 1 & 2 & 2 & 1 & 3 & \emptyset & \emptyset & \emptyset & \emptyset & \emptyset & \emptyset \\ 2^{2} & 1 & \emptyset & 1 & 2 & 3 & \emptyset & 10 & 2 & \emptyset & \emptyset & \emptyset & \emptyset & \emptyset \\ 3^{3} & \emptyset & \emptyset & \emptyset & 1 & 1 & \emptyset & 3 & \emptyset & \emptyset & \emptyset & \emptyset & \emptyset & \emptyset\end{array}$

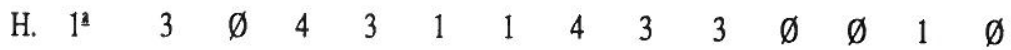
$\begin{array}{llllllllllllll}2^{\mathrm{a}} & 5 & 2 & \emptyset & 4 & 1 & 1 & 9 & 6 & 3 & \emptyset & \emptyset & \emptyset & \emptyset\end{array}$

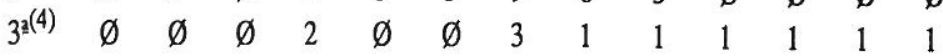

Por orden corresponden a: Nadie, todos (igual), Málaga, Cádiz, Ceuta, Córdoba, los que «no saben» - «cada uno su deje», Sevilla, Granada, Huelva, Jaén, Almería, «parte Sur».

Ie. Si tuvieras que hablar en público, ¿hablarías en andaluz?

SI NO Depende

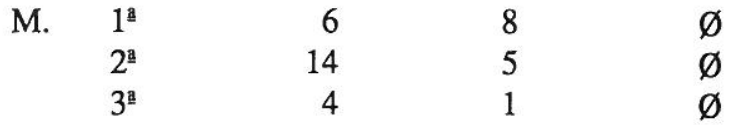


H. $\begin{array}{rrrr}1^{\mathrm{a}} & 10 & 12 & \varnothing \\ 2^{\mathrm{a}} & 21 & 9 & \varnothing \\ 3^{\mathrm{a}} & 7 & 3 & \varnothing\end{array}$

(Se ha añadido el valor vertical «Depende» para el estudio comparativo posterior entre los dos modelos).

IIa. ¿Cómo llamarías a tu forma de hablar?

\begin{tabular}{|c|c|c|c|c|c|}
\hline & Español & Castellano & Andaluz & Ceutí & And-Ceu ${ }^{(5)}$ \\
\hline M. 1 & 1 & 1 & 8 & 2 & 1 \\
\hline $2^{\mathrm{a}(6)}$ & 3 & 2 & 7 & 5 & 3 \\
\hline $3^{\mathrm{a}}$ & $\varnothing$ & $\varnothing$ & 4 & $\varnothing$ & $\varnothing$ \\
\hline H. $1^{\mathrm{a}}$ & 4 & 5 & 9 & 2 & $\varnothing$ \\
\hline $2^{\mathrm{a}}$ & 3 & 5 & 16 & 5 & 1 \\
\hline $3^{a(7)}$ & $\varnothing$ & 1 & 7 & 1 & $\varnothing^{(8)}$ \\
\hline
\end{tabular}

IIb. Crees que tu forma de hablar es CORRECTA o INCORRECTA. ${ }^{(9)}$

\begin{tabular}{|c|c|c|c|c|c|}
\hline & CCR & INCCR & $i ?$ & Mejorable & Normal \\
\hline M. 1 & 9 & 4 & $\varnothing$ & 1 & $\varnothing$ \\
\hline $2^{\mathrm{s}}$ & 10 & 5 & $\varnothing$ & 4 & $\varnothing$ \\
\hline $3^{a}$ & 2 & 3 & $\varnothing$ & $\varnothing$ & $\varnothing$ \\
\hline H. $1^{\mathrm{a}}$ & 17 & 2 & $\varnothing$ & 2 & 1 \\
\hline $2^{a}$ & 19 & 7 & 1 & 2 & 1 \\
\hline $3^{3}$ & 7 & 1 & 1 & 1 & $\varnothing$ \\
\hline
\end{tabular}

IIc. ¿Dónde crees que se habla más correctamente?

d. Provincia andaluza de habla más cuidada, más correcta.

Nd Td Mlg Ceu i? Cdz Cs $\mathrm{Hu}$ Se Gr Alm Cor Jn Sur

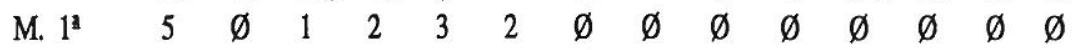
$\begin{array}{lllllllllllllll}2 & 1 & \emptyset & 1 & 3 & 9 & 2 & 1 & \emptyset & 2 & \emptyset & \emptyset & \emptyset & \emptyset & \emptyset\end{array}$

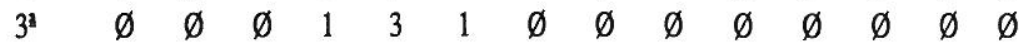

$\begin{array}{llllllllllllllll}\text { H. } & 1 & 3 & \emptyset & 4 & 1 & 4 & 3 & \emptyset & \emptyset & 3 & 3 & 1 & 1 & \emptyset & \emptyset\end{array}$ $\begin{array}{lllllllllllllll}2 & 4 & 1 & \emptyset & 1 & 8 & 4 & 1 & \emptyset & 6 & 3 & \emptyset & 1 & \emptyset & \emptyset\end{array}$ $\begin{array}{lllllllllllllll}3^{\mathbf{a}} & \emptyset & \emptyset & \emptyset & \emptyset & 3 & 2 & \emptyset & 1 & 1 & 1 & 1 & \emptyset & 1 & 1\end{array}$ 
IIe. Si salieras de Andalucía, ¿cambiarías voluntariamente tu modo de expresarte, tu pronunciación?

SI NO Depende

\begin{tabular}{|c|c|c|c|c|}
\hline \multirow[t]{3}{*}{ M. } & $1^{\underline{a}}$ & 2 & 12 & $\varnothing$ \\
\hline & $2^{\mathrm{a}}$ & 2 & 17 & $\varnothing$ \\
\hline & $3^{\mathrm{a}}$ & $\varnothing$ & 4 & 1 \\
\hline \multirow[t]{3}{*}{$\mathrm{H}$. } & $1^{\mathbf{a}}$ & 2 & 20 & $\varnothing$ \\
\hline & $2^{\underline{a}}$ & 3 & 25 & 2 \\
\hline & $3^{\underline{\underline{a}}}$ & 4 & 6 & 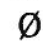 \\
\hline
\end{tabular}

(1) Uno de los entrevistados era bilingüe, castellano y árabe -dijo-; y en IIa. manifestó que su modo de hablar es ceutí, después de reconocer que su hablar no era correcto.

(2) Uno afirmaba con fuerza: «En todos sitios se habla bien menos en Cataluña»

(3) En este apartado hay quien cita dos casos, de ahí que la suma total sea mayor que el número de individuos. En este caso, Valladolid y Castilla.

(4) En una ocasión, un encuestado dijo que se hablaba bien en todas las capitales de provincia.

(5) Se han omitido las excepciones: Norte 1 (1 $\left.{ }^{\mathrm{a}} \mathrm{M}.\right)$, Extremeño 1 ( $\left.3^{\mathrm{a}} \mathrm{M}.\right)$, Canario $1\left(2^{\mathrm{a}} \mathrm{H}\right.$.), Normal $1\left(1^{\mathrm{a}} \mathrm{H}\right)$.

(6) Alguien dijo Español, rectificó ceutí, y luego recalcó los dos. Se han contabilizado los dos.

(7) El caso de un madrileño que cumplía el servicio militar allá, no se ha contabilizado.

(8) El andaluz-ceutí se añadió por no ser caso aislado. Otro encuestado no se definía por ninguna posibilidad, y puntualizó: «cada uno su habla propia».

(9) Se han añadido los valores verticales: $¿$ ? (el que «no sabe», «cada cual», «según» y «depende») el «Mejorable» y el «Normal». Esto ha sido así como expresión o recurrencia para evitar lo directo y eludir la dureza de INCORRECTO.

(10) Se han unificado porque en la localidad encuestada, esta diferenciación era redundante. Un caso que en IIc. dijo Ceuta al preguntarle provincia se vio obligado a nombrar Cádiz. 
Ia $\begin{array}{lllll}\text { M } & \text { Español } & 60,53 \% & \text { Castellano } & 39,47 \% \\ \text { H } & \text { Español } & 48,39 \% & \text { Castellano } & 51,61 \%\end{array}$

$\mathrm{Ib}$

$\begin{array}{llllll} & \text { Bien } & \text { Mal } & \text { Regular } & \text { Normal } & \text { Según } \\ \text { M } & 28,95 \% & 50,00 \% & 7,89 \% & 7,89 \% & 5,26 \% \\ \text { H } & 37,1 \% & 30,64 \% & 24,2 \% & 3,23 \% & 4,83 \%\end{array}$

Ic (1)

$\begin{array}{lllllc} & \text { Ln } & \text { Slm } & \text { Vll } & \text { Ctr } & \text { Ceut } \\ \text { M } & \varnothing & 2,63 \% & 21,06 \% & 13,16 \% & \varnothing \\ \mathrm{H} & 4,84 \% & 1,61 \% & 12,9 \% & 1,61 \% & 3,23 \%\end{array}$

$\begin{array}{llllll} & \text { Mdd } & \text { Cast } & \text { Nit } & \text { And } & \text { ¿? } \\ \text { M } & 21,06 \% & 23,68 \% & 5,26 \% & 5,26 \% & 5,26 \% \\ \text { H } & 16,13 \% & 29,03 \% & 1,61 \% & 6,45 \% & 8,06 \%\end{array}$

Id (2)

$\begin{array}{llllll} & \text { Mlg } & \mathrm{Cdz} & \text { Ceut } & \text { Crd } & \text { Svll } \\ \text { M } & 5,26 \% & 13,16 \% & 15,79 \% & 2,63 \% & 5,26 \% \\ \text { H } & 6,45 \% & 14,5 \% & 3,22 \% & 3,22 \% & 16,12 \%\end{array}$

Ie

$\begin{array}{cccc} & \text { SI } & \text { NO } & \text { i? } \\ \text { M } & 63,16 \% & 36,84 \% & \varnothing \\ H & 61,3 \% & 38,7 \% & \varnothing\end{array}$

(1) Cas, Mch, Sur, Zam, Extr: 1,61\% H ; Tl: 2,63\% M

(2) Gm: $11,29 \% \mathrm{H}$; Alm: 3,22\% H ; Hlv, Alm, Sur: $1,61 \% \mathrm{H}$; $i$ ?: $42,10 \% \mathrm{M}$ y $25,8 \% \mathrm{H}$.

\begin{tabular}{|c|c|c|c|c|c|}
\hline IIa (1) & And & Cast & Esp & Ceu & Abd-Ceu \\
\hline M & $50 \%$ & $7,8 \%$ & $10,4 \%$ & $18,4 \%$ & $10,4 \%$ \\
\hline $\mathrm{H}$ & $51,7 \%$ & $17,75 \%$ & $11,3 \%$ & $13 \%$ & $1,61 \%$ \\
\hline
\end{tabular}




\begin{tabular}{|c|c|c|c|c|c|c|}
\hline \multirow[t]{3}{*}{$\mathrm{Ilb}$} & & Normal & Correcta & Incor & Mejorable & ¿? \\
\hline & M & $\varnothing$ & $55,26 \%$ & $31,58 \%$ & $13,16 \%$ & $\varnothing$ \\
\hline & $\mathrm{H}$ & $3,23 \%$ & $69,35 \%$ & $16,13 \%$ & $8,06 \%$ & $3,23 \%$ \\
\hline \multirow[t]{6}{*}{ IIc-c } & & Nadie & Todos & $i ?$ & Ceut & Svll \\
\hline & M & $15,78 \%$ & $\varnothing$ & $39,5 \%$ & $15,76 \%$ & $5,26 \%$ \\
\hline & $\mathrm{H}$ & $11,29 \%$ & $1,6 \%$ & $24,19 \%$ & $3,22 \%$ & $16,12 \%$ \\
\hline & & Mlg & $\mathrm{Cdz}$ & Cast & Crd & \\
\hline & M & $5,26 \%$ & $13,16 \%$ & $2,63 \%$ & $2,63 \%$ & \\
\hline & $\mathrm{H}$ & $6,45 \%$ & $14,51 \%$ & $1,6 \%$ & $3,22 \%$ & \\
\hline \multirow[t]{3}{*}{ Пе } & - & SI & NO & ¿? & & \\
\hline & M & $10,53 \%$ & $86,84 \%$ & $2,63 \%$ & & \\
\hline & $\mathrm{H}$ & $14,52 \%$ & $82,26 \%$ & $3,22 \%$ & & \\
\hline
\end{tabular}
(1) Canr: $1,61 \% \mathrm{H}$; Nrt, Extr: $2,5 \% \mathrm{M}$
(2) Hlv, Jn, Sur: $1,61 \%$ H ; Alm: 3,22\% ; Grn: $11,29 \%$ 